\title{
System identification analysis for an air compressor system and enhancement proposal by sensors based in nanostructures
}

\author{
Jesus A. Calderón ${ }^{1,2, *}$, Cesar J. Valdivia ${ }^{2}$, Roland Mas $^{2}$, Luis Chirinos ${ }^{2}$, Enrique Barrantes ${ }^{2}$, John H. Lozano ${ }^{2,3}$, \\ Juan $C$. Lengua ${ }^{2}$ \\ ${ }^{1}$ TU Ilmenau University of Technology, Institute of Physics and IMN MacroNano, Germany \\ ${ }^{2}$ Pontificia Universidad Católica del Perú Energy Laboratory and Mechatronic Departament, Perú \\ ${ }^{3}$ Northen (Artic) Federal University named after MV. Lomonosov, Arkhangelsk, Russia.
}

\begin{abstract}
An Air Compressor System is an equipment (mechanical system) which can transmit energy due to increase air pressure level of air flow that through it. This energy can be used in many applications, such as in drills for mining, adding air to tires on vehicles, spraying crops, etc. Therefore, it is necessary to know the mathematical model of an Air Compressor System to study all the thermodynamical variables: "Temperature, Pressure, Air Flow" which let to get a formalized explanation of the energy transfer through this equipment. Furthermore it is described that Air Compressor System can be enhanced while it uses sensors/actuators based in nanostructures.
\end{abstract}

\section{Introduction}

Thermodynamic systems analysis are helpful support to study many machinery, such as for example an air compressor system transforms mechanical energy provided through a motor that connected with a compressor can change pressure level and flow rate on air which is stored inside a tank. Using one scape valve joined on the air tank, temperature variable is correlated with its pressure and air flow changes. Therefore, it can be studied these thermodynamical variables correlations in order to find solutions to many tasks like on medical devices which need faster drills controlled taking action on air compressor systems. It means, it is so important and necessary to know the behaviour of thermodynamical variables on systems, their physical parameters, which can be obtained by "Systems Identification".

However, many times, Systems Identification can't get real approximations of all thermodynamic variable's behaviour on the system, it can be caused by many troubles, like disturbances from vibrations, not expected conditions on the system in operation or also imbalances among impedance between sensors/actuators with system. For this research it was studied a PLINT air compressor system, furthermore, it was analyzed the necessity to replace traditional sensors by sensors based on nanostructures in order to get fast and robust response when the air compressor system is under disturbances or fast changes because of "vibrations, valve operation on the air tank, or non-coordinated delays given by priorities of the Operating System which execute the main algorithm to read every thermodynamic variables of the system.

\section{Theoretical descriptions}

The PLINT air compressor system of Energy Laboratory from Pontificia Universidad Católica del Perú is an educational system designed with a DC motor, an air compressor, air camera, valves, electronic monitoring system, temperature sensors, pressure sensors, and flow sensor. This system did not receive all thermodynamical variables on time, also while could be possible to read physical variables of the process, it was under disturbances like vibrations of impedance among sensor/actuators with hardware interface. For this reason, it was necessary to design a software interface to get interaction between the system due to read thermodynamic variables: Temperatures on compressor, air pressure inside tank, air flow throw tubes connected with the air's tank while it was changed valve's stage it because to look for a non-controlled pressure steady state, owing to study the thermodynamic behaviour of the system; therefore, it was achieved a software interface with an external controller with capacity to operate in Real Time, nevertheless, the connection with an external computer to show thermodynamical process to users needed an Operating Systems from this external computer, that is given by "Windows non real time" so, it did not helped to analyze delays between interactions, special under presence of disturbances. However, this trouble was solved with strategies in the main algorithm

Corresponding author:alan.calderon@pucp.edu.pe 
by an adaptive identification system joined with an observer to monitory information.

By other side, these strategies imply costing by software or by hardware, for this reason, in this work it was studied the advantages obtained while traditional sensors can be replaced through sensors based on nanostructures; owing to these sensors give fast response time and robustness while air compressor system is working. It was studied mathematical model for temperature and pressure sensor based on nanostructures which have Anodic Aluminium Oxide (AAO) [1] as main templates[2], therefore it was possible to identify physical parameters of the system that gave more close results to real parameters, furthermore it was evaluated that a polytropic equation (theoretical analysis for the air flow) can be achieved experimentally with more precision to reality and monitoring data process, which was more stable under disturbances without computing costing. It means, these strategies can help to main processor to solve more complicated tasks than prioritize delays coordination, with these results a system is more efficient, with support to reduce environment contamination to reduce energy consumes and to optimize its operating process[3][4].

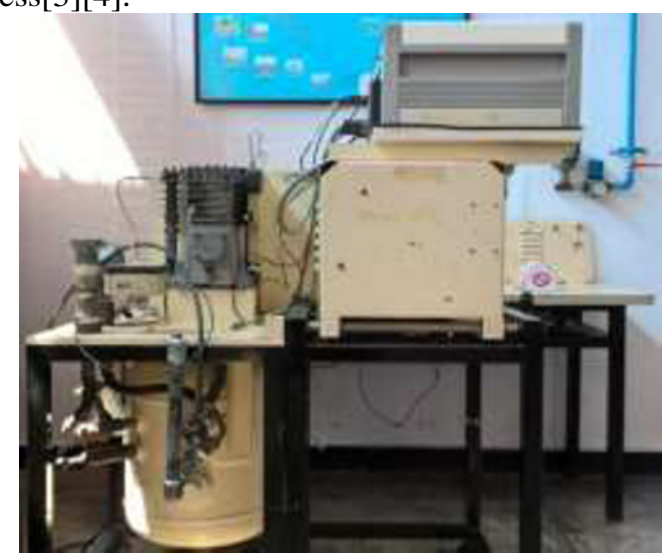

Fig 1. Plint, air compressor thermodynamic system.

It is described in following chapters all the responses excitation signal to the thermodynamical variable of the Air Compressor system, in order to find the mathematical equations for the system, it was studied many methodologies such as "Siegler and Nichols, ARX, Modulating Functions [5], Least Mean Square (LMS)". Nevertheless, due to the system did not has wide nonlinear ranges, even though disturbances, it was identified the system through a hybrid Ziegler and Nichols method (adaptive coefficients with error observer). [6],[7],[8]

\subsection{Relation between torque and input variable}

The input excitation variable is an electrical signal (in Voltage) but this is an auxiliary variable among mechanical movement over a knob with the Revolution Per Minute (RPM) on the DC motor which transmits energy to the compressor system; nevertheless, we studied this signal as input variable because of computer registration. In figure 2 is shown the response of the Air Compressor system because of the input excitation signal, for this context the response was the "Torque variable". As it is depicted there was found linear approximation for the range of work between $1.5 \mathrm{Nxm}$ to $3.2 \mathrm{Nxm}$.

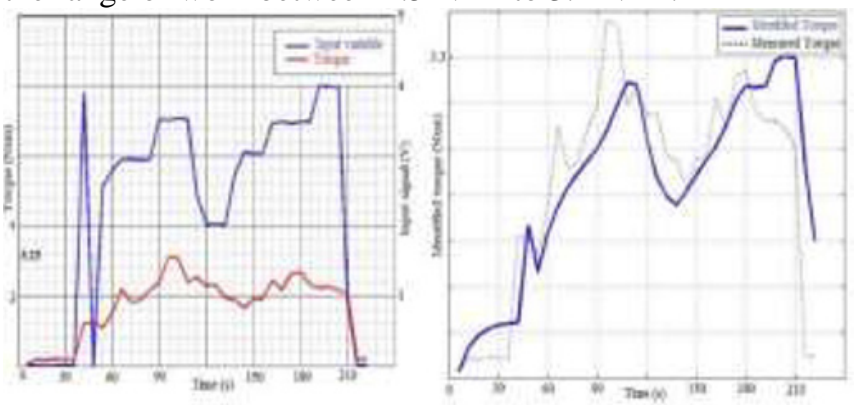

Fig. 2. Input variable versus torque.

For this reason, it was possible to find the transfer function to describe the relation between "Torque and Input Variable (IV) in Laplace (S)" as it is described through equation 1, inside the linear range of work indicated above. It is possible to verify that "Torque's" response time because of "IV" was 3.1 seconds, that value is important while it is necessary to propose a controller of the system in order to correlate necessary torque to be used by the compressor through motor controlling.

$$
\frac{\operatorname{Torque}(S)}{I V(S)}=\frac{0.023}{3.1 S+1}
$$

\subsection{Relation between RPM and input variable}

For the second case, it was studied the Revolution Per Minute "RPM" as a response from the "IV", which is depicted in figure 3, for the linear range of work between 1000 RPM to 2000 RPM (compressor's speed).
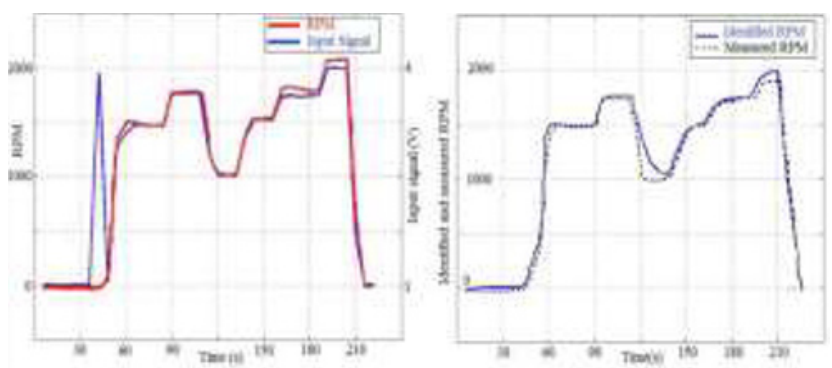

Fig. 3. Input signal versus RPM.

Also, after to look for the static behaviour, it was possible to identify the mathematical model described by equation 2, from which RPM's response time was around 2.8 seconds, that because "IV" can't get instantaneous action over compressor, inertia is present in this dynamic.

$$
\frac{R P M(S)}{I V(S)}=\frac{150}{2.8 S+1}
$$

\subsection{Relation between pressure and input variable}

A very important thermodynamical variable to be studied was "Pressure" of air inside the air's tank, as it is shown 
in figure 4 and its respective identification. From that analysis, itw as simple to verify its response time, 50 seconds, as a consequence of "IV", of course it's so bigger compared with other response time of last variables. This information is so important in order to get thermodynamic analysis and correlations among thermodynamic changes due to mechanical effects.
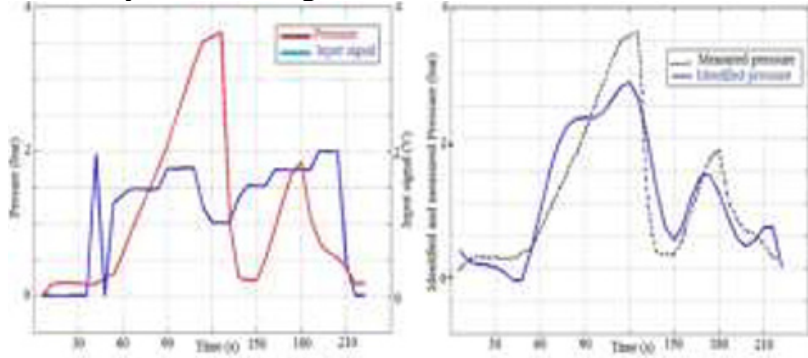

Fig. 4. Input signal versus pressure variable.

$$
\frac{\operatorname{Pressure}(S)}{I V(S)}=\frac{0.68}{50 S+1}
$$

\subsection{Relation between input variable and temperature 1}

For this context, it was assigned as "Temperature 1" to the compressor channel entrance's temperature, in which temperature sensor did not get so bigger changes compared with external temperature, it can be expected due to not high temperature values inside the air tank and external thermal disturbances, that curve is shown and identified in figure 5 . Therefore, it was possible to find the transfer function as described in following equation, from that its response time was around 5.51 seconds
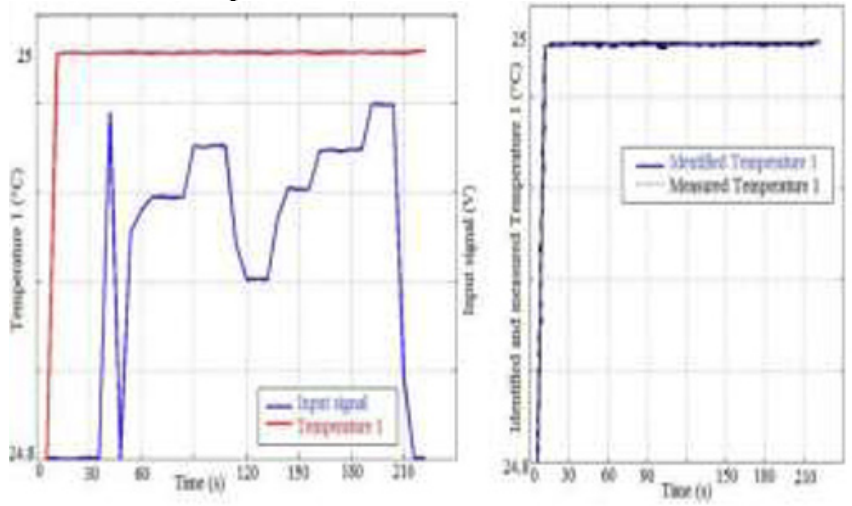

Fig. 5. Input signal versus temperature 1.

$$
\frac{\text { Temperature } 1(S)}{I V(S)}=\frac{0.04}{5.51 S+1}
$$

\subsection{Relation between input variable and temperature 2}

As "Temperature 2", it was considered temperature of air inside the tank, as it can be verified, in figure 6 its experimental curve and its identified curve. From which also is possible to verify its 90 seconds as response time, due to get 93 Celsius degrees from 25 Celsius degrees needs to cross thermal inertia. Therefore, it is very important to get a mathematical equation based in experiments, such as it is described in following equation, from that it can be achieved its dynamic behaviour.
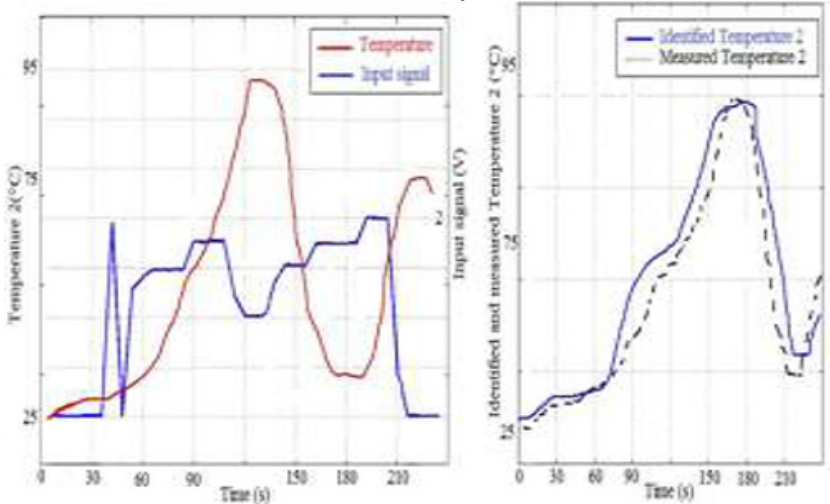

Figure 6. Input signal versus temperature 2.

$$
\frac{\text { Temperature } 2(S)}{I V(S)}=\frac{14}{90 S+1}
$$

\subsection{Relation between input variable and temperature 3}

Air transmission through air tank, connected to its exit, does not get high temperature changes, as it is showed in figure 7. Nevertheless, it is necessary to know that dynamic, which can be obtained from following equation, it is owing to join every partial dynamic of the system.
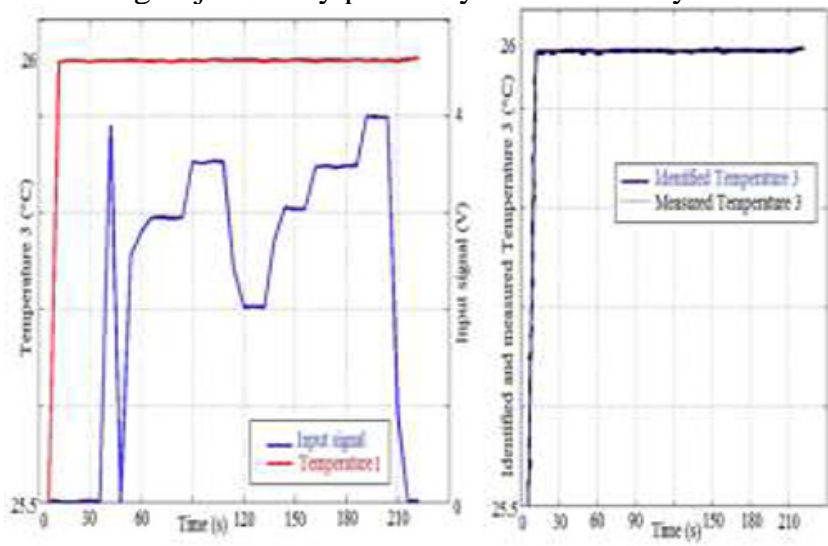

Fig. 7. Input signal versus temperature 3.

$$
\frac{\text { Temperature } 3(S)}{I V(S)}=\frac{0.1}{5.9 \mathrm{~S}+1}
$$

\subsection{Relation among input variable and flow}

In order to analyze behaviour of air flow, when it is open/closed valve (in air tank's exit) it was measured and identified this variable, as it is depicted in figure 8. From these curves was possible to get the mathematical model as described in following equation, which can give information that response time of this variable is so short compared as pressure changes inside air tank and more short time as comparisson with temperature inside the tank. 

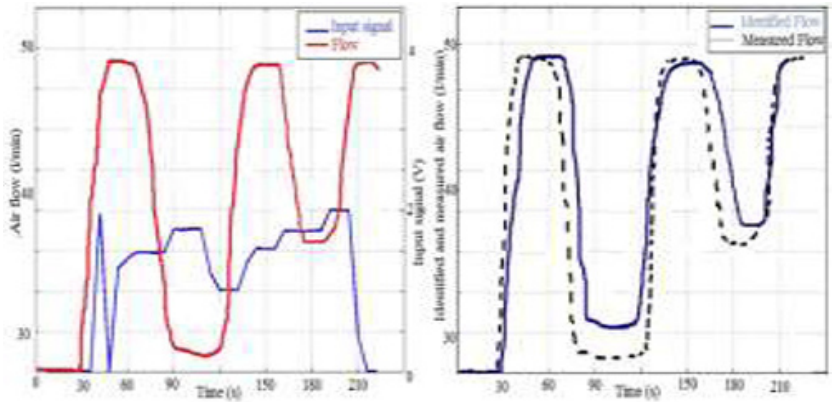

Fig. 8 Input signal versus flow.

$$
\frac{\operatorname{Flow}(S)}{I V(S)}=\frac{9.8}{7 \mathrm{~S}+1}
$$

\section{Systems identification for a new sensor proposal}

In this work was analyzed system identification for thermal properties of an air compressor, from which in figure 9 is depicted as "Temperature 2" in time domain two curves, one of them was obtained through experimental information (curve in color blue) and the other was after to analyze temperature sensor based in nanostructures (curve in color green). It was analyzed physical properties changes for every test as described by authors, from which was possible to get static and dynamic behaviour of sensors/actuators based in nanostructures as dependence of their material and geometry (owing to its high order array) [12], [13], [14].

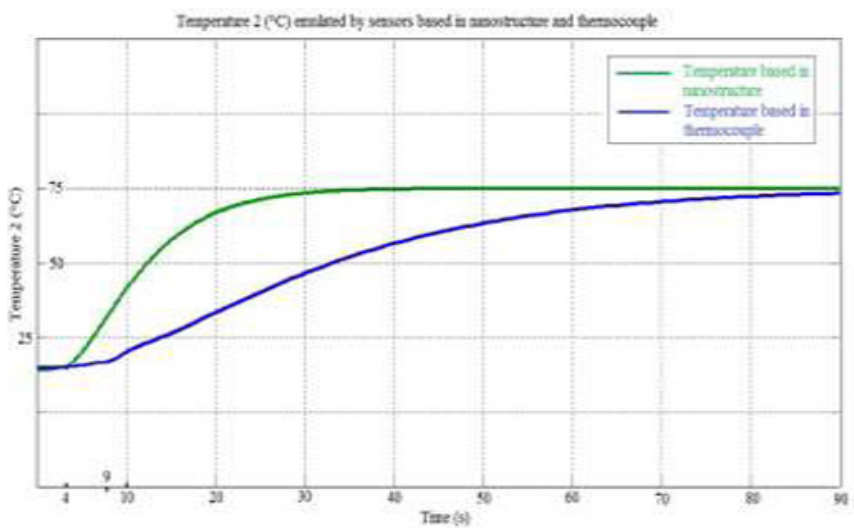

Fig. 9. Temperature sensors comparison.

From which, can be possible to find transfer functions and also to verify response time from both proposal sensors. Therefore, while the input excitation is an electrical voltage through a step with amplitude $5 \mathrm{~V}$, therefore the Transfer Function for a classic thermocouple type $\mathrm{K}$ is given by equation:

$$
\frac{\operatorname{Temp}_{\operatorname{TypeK}}(S)}{\operatorname{InputV}(S)}=\frac{11}{70 S+1} \mathrm{e}^{-9 S}
$$

The expression in time domain is given through the equation, that is obtained from last equation after to
$70 \frac{\mathrm{d} T e m p_{\text {турек }}(t)}{\mathrm{d} t}+\mathrm{Temp}_{\text {турек }}=11 \Delta \mathrm{U}(t)$

From which, the solution is

$$
\operatorname{Temp}_{\text {TypeK }}(\mathrm{t})=55(1-\exp (-0.014 t))
$$

By other side, the Transfer Function obtained for the proposal sensor based in nanostructures is given by equation

$$
\frac{\operatorname{Temp}_{\text {nano }}(\mathrm{S})}{\operatorname{InputV}(S)}=\frac{11}{20 S+1} \mathrm{e}^{-4 S}
$$

The expression in time domain is given through the equation, that is obtained from last equation after to

$$
20 \frac{\mathrm{d} T e m p_{\text {nano }}(t)}{\mathrm{d} t}+\operatorname{Temp}_{\text {nano }}(t)=11 \Delta \mathrm{U}(t)
$$

From which, the solution is

$$
T e m p_{\text {nano }}(\mathrm{t})=55(1-\exp (-0.05 t))
$$

\section{Comparisons}

It has been made experiments by an Air Compressor System, in which was tested by traditional sensors the thermodynamic variables of the system "pressure, temperature and flow", furthermore these results were compared by simulations of nanosensors according to propose that nanosystems can be connected with macrosystems and also to give robustness to system operations such as is described in this work, that is shown in identification model results through thermodynamic analysis of this work.

From equation to describes output pressure of air compressor it is found its solution as general represented in equation, however the analysis was made by nanostructures dynamic analysis described in transfer functions above.

$$
\mathrm{a}_{1} \frac{\mathrm{d} P(t)}{\mathrm{d} t}+\mathrm{a}_{2} P(t)=\mathrm{a}_{3} \Delta \mathrm{U}
$$

Its solution proposal is given by

$$
\mathrm{P}(\mathrm{t})_{2}=(1-\exp (t)) \Delta \mathrm{U}
$$

Similar case for flow

$$
\mathrm{b}_{1} \frac{\phi(t)}{\mathrm{d} t}+b_{2} \phi(t)=\mathrm{b}_{3} \Delta \mathrm{U}
$$

In similar context, its solution proposal is given by

$$
\phi(t)=(1-\exp (\mathrm{t})) \Delta \mathrm{U}
$$

therefore, taking "ln" in pressure and flow equations, it is obtained following equation due to it is a constant (input excitation signal) that joined both

$$
\ln P(t)+\mathrm{K} \ln \phi(\mathrm{t})=\Delta \mathrm{U}
$$

It means 


$$
\mathrm{P}(t) \phi(t)^{K}=\Delta \mathrm{U}
$$

Therefore, for air polytropic index around 1.4, from which were obtained 2 values for " $K$ ", that depends of " $a_{1}, a_{2}, a_{3}, b_{1}, b_{2}, b_{3}$ ". It means that it was obtained polytropic index value near 1.4 as compared with experimental data from this work (in which was not used sensors based in nanostructures).

\section{Conclusions}

In this work it has been determined mathematical equations for a Thermodynamic System (an air compressor) through "Systems Identification" analysis. Moreover, it has been compared by simulations, the performance in this system among traditional sensors with sensors based in nanostructures, which have robustness and a short response time, it means a good consequence to save energy and to avoid environment consequences.

Furthermore, there is expressed kind greetings to Mr. Broni Huamaní, Mr. Eduardo Pichilingue, Mr. César José Leclere Mota and Mr. Bruno Sebastián Miranda Quispe because of all their support during experimental tests.

\section{References}

1. Seo-hyeon Jo (2012). Fabrication and crystalization of AAO template for sensor applications.

2. Hongdan Yan (2012). Preparation and optical characterization of nanoporus templates as a basis for nanocontact arrays.
3. Yong Lei (2007). Higly ordered nanostructures with tunable size, shape and properties: A new way to surface nano-pattering using ultra-thin alumina masks.

4. Honeywell (1998). Megopal Thermocouples, sepecification.

5. Lennart Ljung (1994). Modeling of dynamic systems.

6. Liuping Wang (2009). Model Predictive Control System design and implementation using MATLAB.

7. Pearson A. E. (1995). Aerodynamic Parameter Estimation Via Fourier Modulating Function Techniques.

8. Klamka, Jerzy (2008). Controllability of dynamical systems.

9. CompactRio National Instruments. http://www.ni.com/datasheet/pdf/en/ds-201.

10. MyRio National Instruments. http://www.ni.com/pdf/manuals/376047a.pdf.

11. Monolithic Thermocouple Amplifiers with Cold Juntion Compensation AD595, Analog Devices.

12. Il Seo (2009). Assembly of Colloidal Nanoparticles into Anodic Aluminum Oxide Templates by DipCoating Process.

13. Nanolike, temperature nanosensor. www.sensortest.de/ausstellerbereich/upload/mnpdf/en/Nanolikete mperaturenanosensors15.pdf

14. Yong Lei (2006). High ordered nanostructures with tunable size, shape and properties: A new way to surface nanopattering using ultrathin alumina masks. 